\title{
Aromatics adsorption properties onto oxidized activated carbon from aqueous solution
}

\author{
Motoi Machida**, Mariko Hayashida*, Yuichi Kato** and Hideki Tatsumoto*
}

\begin{abstract}
Adsorption of various aromatics onto activated carbons oxidized by nitric acid in aqueous solution was examined comparing with non-oxidized counterparts. The aromatics used were aniline, benzoic acid, nitrobenzene, phenol and bisphenol A. All aromatics exhibited two-step adsorption isotherms for oxidized activated carbons with the rise in concentration of adsorbates, whereas only one-step adsorption isotherms were observed for non-oxidized carbons. The amount of aromatics adsorption was also decreased by oxidation except bisphenol A. The aromatics adsorption sites on the oxidized activated carbon were considered to be different from those on the non-oxidized carbon. Adsorption mechanism was investigated by a brief numerical analysis for the experimental data of two-step adsorption isotherms principally assuming a two-layer adsorption model and a mono-layer reorientation model. Based on the analysis for the two-step isotherms observed on the oxidized activated carbon, a reorientation model switching from flat-on to end-on molecular position is probable as well as a two-layer model.
\end{abstract}

\section{KEYWORDS : Activated carbon, Oxidation, Adsorption, Adsorption properties, Surface oxygen complexes}

\section{Introduction}

Environmentally persistent synthetic organic compounds are threat to human beings as well as ecosystem. Harmful effects are frequently observed when they are biologically accumulated in a living body via food chain in water environment. Some of them such as nonylphenol and bisphenol A also work as environmental endocrine disruptors by very low concentration ${ }^{1)}{ }^{2)}$. Adsorption to activated carbon in aqueous solution is a widely used attractive option for removing the hazardous organic compounds. Activated carbon is a porous material resulting in a large specific surface area of more than $1000 \mathrm{~m}^{2} / \mathrm{g}$. Adsorption characteristics of activated carbon are influenced by not only surface area, but also pore distribution and surface functional groups to much extent. From our previous study and the literature, heavy metals adsorption was greatly enhanced by the introduction of acidic oxygen functional groups such as carboxyl and lactone ${ }^{3), 4)}$, whereas aromatics adsorption were significantly reduced in the presence of surface carbon-oxygen complexes $\left.{ }^{5)}, 6\right)$. When phenol adsorbed onto an oxidized activated carbon comparing with a non-oxidized one, the amount of phenol adsorption onto the oxidized carbon was less than $20 \%$ of the non-oxidized at a lower initial phenol concentration, but at a higher phenol concentration adsorption capacity onto oxidized carbon was increased depicting two-step shaped isotherm up to more than $65 \%$ of non-oxıdized carbon ${ }^{7)}$. In spite of the fact that such a two-step adsorption isotherm for phenol adsorption onto oxidized carbon had already been reported more than half a century $\mathrm{ago}^{8)}$, the adsorption mechanism for the two-step isotherm was not clearly elucidated. Two- or multi-layer model including B. E. T. model ${ }^{9)}$, reorientation model switching from flat-on to end-on position of adsorbed molecules, and capillary condensation model were suggested for the two-step adsorption isotherms ${ }^{10)}$, 11). The only fact experimentally proven is that the phenol adsorption sites of the oxidized activated carbon were different from those of the non-oxidized ${ }^{7)}$. The objective of the present study, therefore, is to discuss the adsorption mechanism of the two-step isotherms observed on oxidized carbons in aqueous solution using several kinds of aromatics adsorption combining with a brief numerical analysis of the resultant adsorption isotherms data.

\section{Materials and methods}

\subsection{Adsorbents and their treatments}

The carbonaceous adsorbents used were two commercially available granular activated carbons (GAC) made from coconut shell (Calgon Mitsubishi Chemical Corporation, Diasorb W10-30) and coal (Calgon Corporation, Filtersorb 400). The activated carbons were washed in boiling de-ionized water repeatedly until no further $\mathrm{pH}$ change could be observed, and then dried in air oven at $383 \mathrm{~K}$ until 
any decrease in amount was not measured. The dried GAC's were used as non-oxidized activated carbons ${ }^{12)}$. The coconut shell based GAC was referred to as GAC-1, and coal based was as GAC-2 in the study. The GAC-1 and GAC-2 were oxidized with 7.5 $\mathrm{M} \mathrm{HNO}_{3}$ solution controlling temperature at $363 \mathrm{~K}$ for 6 hours, and washed with de-ionized water repeatedly until no further change in aqueous solution $\mathrm{pH}$ was detected as well, dried in air oven at $383 \mathrm{~K}$ followed by calcining at $623 \mathrm{~K}$ to completely remove nitrate ion remained in the GAC's, and finally allowed to cool in desiccator. The oxidized GAC's were referred to as Oxi-GAC-1 and -2 for coconut shell and coal based, respectively. Oxi-GAC-1 was subsequently out-gassed at $1273 \mathrm{~K}$ in helium flow for 1 hour ${ }^{13)}$, denoting Otg-GAC-1.

Specific surface areas of the adsorbents were measured by B. E. T. method using $\mathrm{N}_{2}$ adsorption isotherms at $77 \mathrm{~K}$ with a surface analyzer (Beckman Coulter Model SA3100). The total surface area which is sum of micropore and mesopore area, and mesopore area were also calculated by $t$-plot method from the $\mathrm{N}_{2}$ adsorption isotherms ${ }^{14)}$; the micropore surface area was obtained by subtracting the mesopore area from the total area.

Acidic and basic surface functional groups were determined according to the Boehm's method ${ }^{15)}$. One gram of GAC's or $0.7 \mathrm{~g}$ of Ox1-GAC's was placed in $15 \mathrm{~mL}$ of the following $0.1 \mathrm{M}$ solutions : sodium hydroxide, sodium carbonate, sodium bicarbonate, and hydrochloric acid ${ }^{16)}$. The vials were sealed and agitated for more than 4 days and then $5 \mathrm{~mL}$ of each solution separated from the adsorbent was pipetted and the excess of base and acid was titrated with $\mathrm{HCl}, \mathrm{NaOH}, \mathrm{Na}_{2} \mathrm{CO}_{3}$ and $\mathrm{NaHCO}_{3}$, respectively. The number of acidic sites was calculated assuming that $\mathrm{NaOH}$ neutralizes carboxyl, lactonic and phenolic groups ; $\mathrm{Na}_{2} \mathrm{CO}_{3}$ does carboxyl and lactonic ; $\mathrm{NaHCO}_{3}$ only carboxyl groups. The number of surface basic sites was calculated from the amount of $\mathrm{HCl}$ neutralization.

\subsection{Adsorption measurements of aromatics}

Aromatic compounds examined in the study were aniline, benzoic acid, nitrobenzene, phenol and bisphenol A. All aromatics were purchased by commercial regent grade. The stock solution of each aromatic compound was prepared dissolving it with de-ionized water at a desired concentration. The adsorption experiments were carried out with batch systems. Fifty $\mathrm{mL}$ of aqueous solution of the aromatics bearing 50 or $100 \mathrm{mg}$ of adsorbents in a $100 \mathrm{~mL}$ stoppered conical flask was agitated at $100 \mathrm{rpm}$ at ambient temperature. Four days was known to be sufficient period to reach adsorption equilibrium on the system in our preliminary experiments. The equilibrium solution was easily separated from adsorbent using decantation technique because adsorbent was granular form and resistant enough to abrasion under the conditions resulting in no powdered materials in the solution. The aromatics concentration was determined by UV-Vis spectrophotometer (SHIMADZU UV2550) after diluting the solution with hydrochloric acid to adjust $\mathrm{pH}$. The maximum absorption wavelength determined between 241.5 to $275 \mathrm{~nm}$ according to the type of aromatic compound. The amount of adsorbed onto the activated carbons was calculated by difference between initial and final equilibrium concentrations.

\section{Results and discussion}

\subsection{Properties of adsorbents}

Table 1 shows specific surface areas of mesopore and micropore, and surface functional groups for activated carbons, and their oxidized and out-gassed counterparts. The out-gassed carbon is donated Otg-GAC-1. Specific surface areas of the activated carbons are decreased on oxidation caused by converting a part of micropore to mesopore. Activated carbon was manufactured using temperature around $1000 \mathrm{~K}$ in which graphene sheet and stacked layers were estimated to be insufficiently developed including fullerene-like structure ${ }^{17)-19)}$. Surface functional groups introduced by oxidation would be located on the edge of the insufficiently developed graphite sheets, and widen the space of graphite layers. The widened graphite sheets space was reversibly shrunk to the original space by removing surface oxygen functional groups in out-gassing as tabulated in Table 1 ; the Otg-GAC-1 of outgassing Oxi-GAC-1 was obviously demonstrated to be recovered the original surface area and pore distribution.

\subsection{Aromatics adsorption onto non-oxidized and oxidized activated carbons}

Aromatics adsorption isotherms of aniline, benzoic acid, nitrobenzene, phenol and bisphenol A onto non-oxidized activated carbons of GAC-1 and GAC-2, and oxidized counterparts of OxiGAC-1, Oxi-GAC-2 are depicted in Figs.1 through 6. The equilibrium solution $\mathrm{pH}$ was always less than 6.5. It is apparent that oxidation

Table 1 Properties of activated carbons.

\begin{tabular}{rccccc}
\hline & GAC-1 & Oxi-GAC-1 & Otg-GAC-1 & GAC-2 & Oxi-GAC-2 \\
\hline Surface area, $\mathrm{m}^{2} / \mathrm{g}$ & 1000 & 890 & 1030 & 1030 & 710 \\
mesopore, $\mathrm{m}^{2} / \mathrm{g}$ & 190 & 480 & 220 & 160 & 150 \\
micropore, $\mathrm{m}^{2} / \mathrm{g}$ & 810 & 400 & 820 & 860 & 560 \\
Boehm titration, meq./g & & & & & \\
carboxyl groups & 0.03 & 1.14 & 0.00 & 0.03 & 1.80 \\
lactonic groups & 0.04 & 0.04 & 0.06 & 0.02 & 0.07 \\
phenolic groups & 0.23 & 0.23 & 0.18 & 0.16 & 0.04 \\
Total acidic groups & 0.30 & 1.41 & 0.24 & 0.21 & 1.91 \\
Total basic groups/sites & 0.47 & 0.00 & 0.73 & 0.50 & 0.00 \\
\hline
\end{tabular}


greatly reduces the adsorption capacity for all aromatics except bisphenol A, and as can be seen in Table 1, Figs.3 and 5, the more oxygen functional groups, the more are the adsorption capacities decreased. The shape of the isotherms examined seems to be twostep for the aromatics adsorption onto the oxidized activated carbons whereas only one-step isotherms are observed for the nonoxidized activated carbons. Since it is widely recognized that the presence of electron withdrawing groups of carboxyl and lactone greatly reduce the binding function by $\pi$-electrons, aromatics adsorption onto graphene layer will be no longer valid for oxidized activated carbons. As shown in Table 1, the disappearance in basic groups/sites on oxidation is considered to be corresponding to significant decrease in $\pi$-electrons on the graphite surface. Instead, the adsorption sites can be switched from graphene layers to on or near the oxygen functional groups located at the edges of

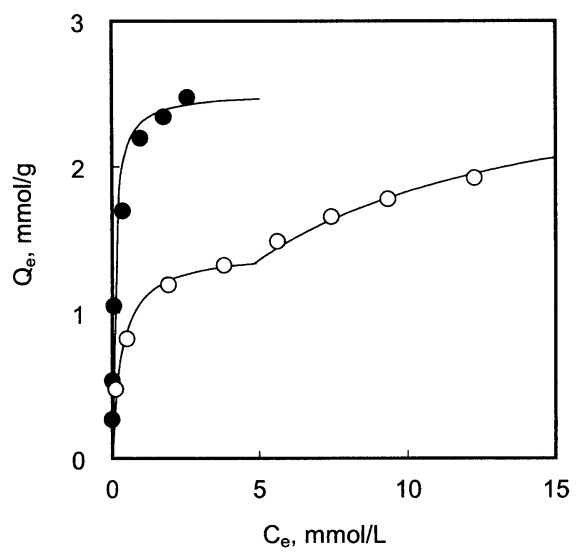

Fig.1 Adsorption isotherms of aniline onto non-oxidized activated carbon (GAC-1 ; O) and oxidized activated carbon (OxiGAC-1 ; O). Solid lines ; predicted by Langmuir parameters assuming reorientation model for the second step of oxidized carbon, dotted line ; extrapolation of the first step isotherm of oxidized carbon.

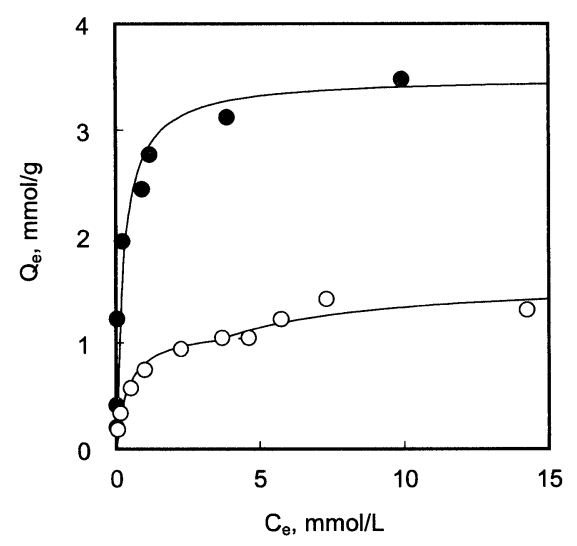

Fig.2 Adsorption isotherms of benzoic acid onto non-oxidized activated carbon $(\mathrm{GAC}-1 ; \mathrm{O})$ and oxidized activated carbon (Oxi-GAC-1 ; O). Solid lines ; predicted by Langmuir parameters assuming reorientation model for the second step of oxidized carbon, dotted line ; extrapolation of the first step isotherm of oxidized carbon. the graphene layers by the oxidation, due to the fact that the rate determining step of phenol adsorption is also found to be changed from diffusion control to adsorbate-surface interactions control requiring shorter adsorption time by the introduction of oxygen functional groups ${ }^{7)}$. When the plots of adsorption conversion against the square root of time are constant in various initial concentrations, the adsorption will be operated by diffusion control mechanism, while it will be adsorption control when the plots are proportional to the initial concentratıons according to the literature by Nevskaia et al. ${ }^{7)}$. For the oxıdized activated carbon, the adsorption is considered to take place at relatively external area of the carbon $^{20)}$, though it would occur in the inner narrow micropores for the non-oxidized carbon ${ }^{21}$ ).

Langmuir parameters assuming the following equation were tabulated in Tables $\mathbf{2}$ and $\mathbf{3}$ for one-step isotherms for GAC- 1 and -2, and the first-step isotherms in two-step isotherms for Oxi-GAC-1 and -2 , respectively.

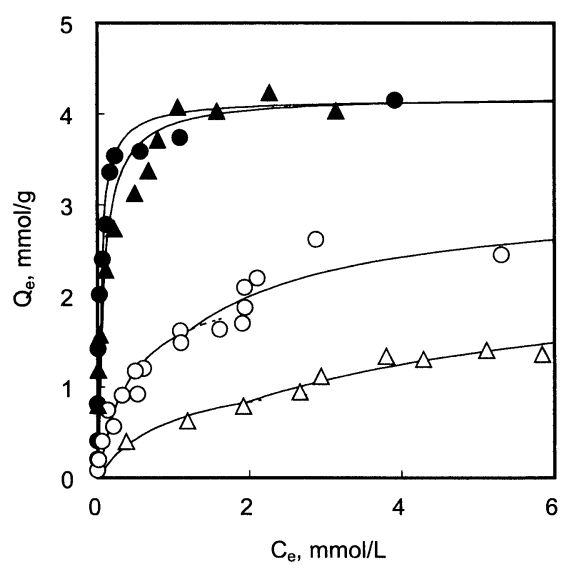

Fig.3 Adsorption isotherms of nitrobenzene onto non-oxidized activated carbon (GAC-1;, GAC-2 ; $\boldsymbol{\Delta}$ ) and oxidized activated carbon (Oxi-GAC-1 ; $\bigcirc$, Oxi-GAC-2 ; $\triangle$ ). Solid lines ; predicted by Langmuir parameters assuming reorientation model for the second step of oxidized carbon, dotted lines ; extrapolation of the first step isotherms of oxidized carbons.

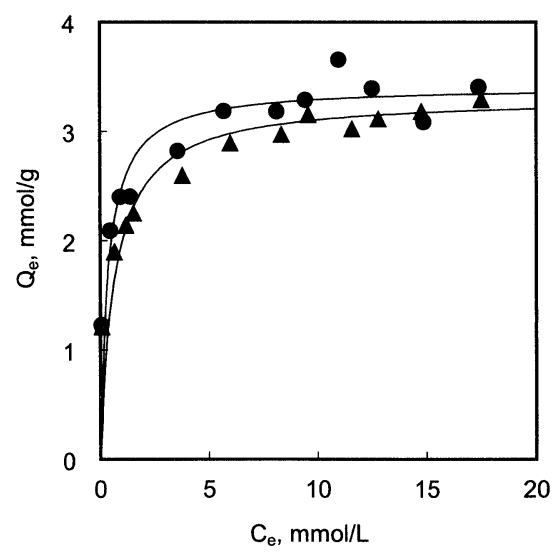

Fig.4 Adsorption isotherms of phenol onto non-oxidized activated carbon (GAC-1 ;, GAC-2 ; $\mathbf{\Delta}$ ). Solid lines ; predicted by Langmuir adsorption parameters. 


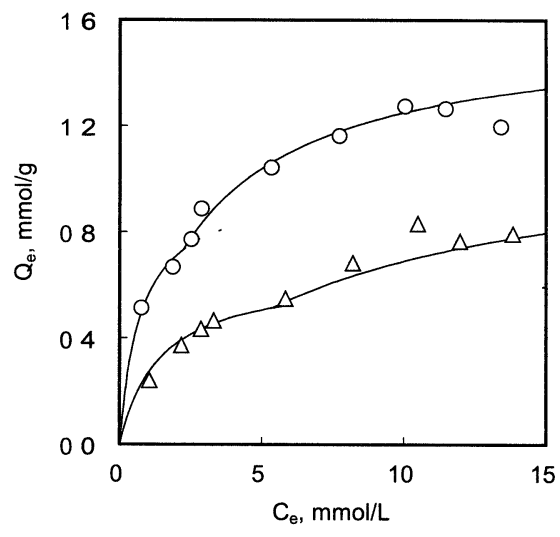

Fig.5 Adsorption isotherms of phenol onto oxidized activated carbon (Oxi-GAC-1 ; O, Oxi-GAC-2 ; $\triangle$ ). Solid lines ; predicted by Langmuir parameters assuming reorientation model for the second step of oxidized carbons, dotted line ; extrapolation of the first step isotherms of oxidized carbons.

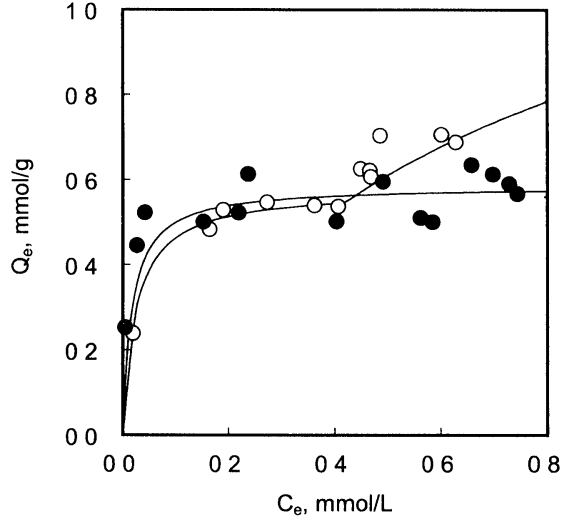

Fig.6 Adsorption isotherms of bisphenol A onto non-oxidized activated carbon (GAC-1; $)$ ) and oxidized activated carbon (Oxi-GAC-1 ; O). Solid lines ; predicted by Langmuir parameters assuming reorientation model for the second step of oxidized carbon, dotted line ; extrapolation of the first step isotherm of oxidized carbon.

Table 2 Langmuir adsorption parameters of various organic compounds for non-oxidized activated carbon.

\begin{tabular}{|c|c|c|c|c|c|}
\hline Adsorbate & Anıline & Benzoic acid & Nitrobenzene & Phenol & Bisphenol A \\
\hline \multicolumn{6}{|l|}{ GAC-1 } \\
\hline$X_{m}, \mathrm{mmol} / \mathrm{g}$ & 2.5 & 3.5 & 4.2 & 3.4 & 0.59 \\
\hline$K_{e}, L / m m o l$ & 11 & 3.8 & 24 & 2.7 & 58 \\
\hline \multicolumn{6}{|l|}{ GAC-2 } \\
\hline$X_{m}, \mathrm{mmol} / \mathrm{g}$ & - & - & 4.2 & 3.3 & - \\
\hline$K_{e}, L / \mathrm{mmol}$ & - & - & 12 & 1.5 & - \\
\hline
\end{tabular}

Table 3 Langmuir parameters for the first-step isotherms in two-step aromatics adsorption onto oxidized activated carbon.

\begin{tabular}{cccccc}
\hline Adsorbate & Aniline & Benzoic acid & Nitrobenzene & Phenol & B1sphenol A \\
\hline Oxi-GAC-1 & & & & & \\
$X_{m}$, mmollg & 1.4 & 1.1 & 2.2 & 0.97 & 0.58 \\
$K_{e}$, L/mmol & 3.1 & 2.4 & 2.2 & 1.4 & 40 \\
Oxi-GAC-2 & & - & 1.2 & 0.65 & - \\
$X_{m}$, mmollg & - & - & 1.1 & 0.70 & - \\
$K_{e}$, L/mmol & - & - & & \\
\hline
\end{tabular}

$$
\frac{C_{e}}{Q_{e}}=\frac{1}{X_{m} K_{e}}+\frac{C_{e}}{X_{m}}
$$

where $C_{e}$ and $Q_{e}$ are the equilibrium concentrations in the solution and on the adsorbent, and $X_{m}$ and $K_{e}$ are the maximum number of aromatics adsorption sites and the adsorption affinity onto the adsorption sites, respectively. The $X_{m}$ and $K_{e}$ can be calculated from the slope and intercept of the approximated straight line when $C_{e} / Q_{e}$ is plotted against $C_{e}$. Both resultant adsorption capacity of $X_{m}$ and adsorption strength onto adsorbent of $K_{e}$ for the non-oxidized activated carbons are greater than those for the oxidized carbons in any aromatic compound used, reflecting stronger $\pi-\pi$ dispersive interactions of aromatics onto the non-oxidized activated carbons, and for oxidized activated carbon forming donor-acceptor complexes between surface oxygen functional groups (electron donors) and the aromatic ring of adsorbate acting as the acceptor ${ }^{22)}$, and/or forming hydrogen-bonds between the oxygen groups and aromatics ${ }^{23)}$. According to the results of Franz et al., phenol and aniline adsorption in the absence of water, instead in the cyclohexane media, was enhanced for the oxidized carbon more than the non-oxidized carbon revealing that hydrogen bonding rather than electrons donor-acceptor mechanism could be working on the oxygen functional groups ${ }^{23)}$.

Since aromatics of aniline, benzoic acid, nitrobenzene and phenol adsorb in mono-layer on the activated carbon surface as can be calculated from $X_{m}$ values, most of the adsorption would take place at micropore in which microporosity effects could play a major role in the adsorption of aromatics ${ }^{24)}$. The $\pi-\pi$ dispersive interactions between $\pi$-electrons of benzene rings of aromatics and those of graphene layer for non-oxidized carbon, therefore, exhibit stronger adsorption affinity than for the oxidized carbons. On the contrary, since bisphenol A has larger molecular size resulting in limited adsorption sites to mesopore and macropore, there will be no major difference between the non-oxidized and the oxidized carbons in the adsorption capacities of $X_{m}$ 's and the adsorption affinities of $K_{e}$ 's calculated from bisphenol A isotherms as shown in Tables 2 and 3. 


\subsection{Two-step aromatics adsorption isotherms by oxidized activated carbons}

As mentioned previously in the introduction, although two-step or two-plateau isotherms for phenol adsorption had been observed more than half a century ago ${ }^{7), 8)}$, the mechanism of two-step isotherms was not clearly elucidated. The first steps are considered to be mono-layer adsorption of aromatics since the Langmuir isotherms can well represent the experimental results as drown in solid lines on Figs.1-3, 5 and 6, as well as one-step isotherms observed for the non-oxidized activated carbon. Although the appearance of the second step adsorption isotherms for the oxidized activated carbons has not been well known, several mechanisms are proposed such as capillary condensation, two-layer and molecular reorientation models. Though capillary condensation as the second step was frequently observed in vapor and liquid phase adsorption to activated carbon ${ }^{25)}$, the shape of isotherms and no plateaus characterizing the capillary condensation was not observed in the experiments, thus the capillary mechanism could be excluded for the present study ${ }^{26)}$. In phenol adsorption, for instance, the two layer and the reorientation mechanisms were suggested for the twostep isotherms ${ }^{11)}$. While the second plateau may represent the second condensed mono layer formed on top of the first for the two-layer model, for the reorientation mechanism, the shape of the adsorption molecules on the carbon surface can be changed from the prostrate flat-on position for the first step isotherms to end-on position for the second step isotherms. In the present study, Langmuir parameters were calculated by the eq. (1) as shown in Table 4 assuming the two layer and the reorientation mechanisms. For the two-layer model assuming that the second layer starts to be formed on top of the first layer adsorption after the first layer is formed by selfassembling via sufficient amount of adsorption ; two monolayer is supposed to be formed to calculate the Langmuir parameters. The calculated adsorption affinities of $K_{e}$ 's to the second layer are less than those to the first layer except some exceptions for benzoic acid and nitrobenzene. The adsorption capacities of $X_{m}$ 's for the second layer are also less than those obtained for the first layer as expected for the model. Therefore, the two-layer model can be valid for the two-step isotherms.

The reorientation mechanism can also be proposed for the twostep isotherms. Aromatic compounds can be adsorbed on the platinum electrodes by both flat-on and end-on position in which the flat-on configuration is predominant to the end-on in lower adsorbate concentration $^{27)}$. In assuming the reorientation model, the adsorption capacities of $X_{m}$ 's for the end-on position are greater than those of the flat-on position for all adsorbates as expected, and adsorption strengths of $K_{e}$ 's are always successfully less than flat-on position as well. The resultant orders support the assumption of the reorientation model. The flat-on/end-on ratio for the occupied area per aromatic molecule were reported ranging 1.5 and $3.0^{27)}$ prosperously consistent with the range of 1.4 to 2.6 of $X_{m}$ 's ratio of $X_{m, e n d-o n} / X_{m, f l a t-o n}$ for the second to the first steps of adsorption isotherms of aniline, benzoic acid, nitrobenzene and phenol as shown in Table 4. Aromatics molecules may be viewed as adsorbed by end-on position with larger concentration bringing about cooperative adsorption owing to interaction of neighboring adsorbate molecules switching from flat-on orientation at lower concentration ${ }^{11)}$. ; vertically oriented close packed monolayer with strong intermolecular forces can be operative between their hydrophobic residues exerting their maximum effect ${ }^{9}$. The solid lines in Figs.1-3, 5 and 6 for the second step isotherms by the oxidized carbons were depicted using the Langmuir parameters of the reorientation model. These results indicated that the reorientation model as well as the two-layer model can be probable mechanism for the two-step isotherms.

Furthermore, Franz et al. pointed out that in the aqueous media water cluster would be formed around the oxygen functional groups via hydrogen bonding leading to blocking the access of aromatics

Table 4 Langmuir parameters for the second-step isotherms in two-step aromatics adsorption onto oxidized activated carbon.

\begin{tabular}{|c|c|c|c|c|c|}
\hline Adsorbate & Aniline & Benzoic acid & Nitrobenzene & Phenol & Bisphenol A \\
\hline \multicolumn{6}{|c|}{$\begin{array}{l}\text { Two-layer model: second-layer adsorption on top of the first-layer adsorption } \\
\left(X_{m}: \text { limited to the second layer adsorption capacity for the two-layer model }\right)\end{array}$} \\
\hline \multicolumn{6}{|c|}{ Oxi-GAC-1 } \\
\hline$X_{m}$, mmollg & 0.85 & 0.30 & 2.0 & 0.35 & 0.17 \\
\hline$K_{e}, \mathrm{~L} / \mathrm{mmol}$ & 0.23 & 2.5 & 1.0 & 0.70 & 29 \\
\hline \multicolumn{6}{|l|}{ Oxi-GAC-2 } \\
\hline$X_{m}, \mathrm{mmol} / \mathrm{g}$ & - & - & 0.37 & 0.25 & - \\
\hline$K_{e}, L / m m o l$ & - & - & 5.5 & 0.60 & - \\
\hline \multicolumn{6}{|c|}{$\begin{array}{l}\text { Reorientation model: end-on adsorption switched from flat-on adsorption for the first step } \\
\left(X_{m}: \text { the total adsorption capacity for the reorientation model }\right)\end{array}$} \\
\hline \multicolumn{6}{|c|}{ Oxi-GAC-1 } \\
\hline$X_{m}, \mathrm{mmol} / \mathrm{g}$ & 2.8 & 1.6 & 3.1 & 1.6 & 1.5 \\
\hline$K_{e}, L / m m o l$ & 0.20 & 0.50 & 0.90 & 0.39 & 1.5 \\
\hline$X_{m, \text { end on }} / X_{m, \text { flat on }}$ & 2.0 & 1.5 & 1.4 & 1.6 & 2.6 \\
\hline \multicolumn{6}{|l|}{ Oxi-GAC-2 } \\
\hline$X_{m}, \mathrm{mmol} / \mathrm{g}$ & - & - & 2.4 & 1.2 & - \\
\hline$K_{e}, L / m m o l$ & - & - & 0.27 & 0.15 & - \\
\hline$X_{m, \text { end on }} / X_{m, \text { flat-on }}$ & - & - & 2.0 & 1.8 & - \\
\hline
\end{tabular}


adsorbates to the adsorption sites ${ }^{23)}$. The water cluster model as an alternative mechanism can, therefore, also explain the experimental results for the oxidized activated carbons for changes in adsorption capacities and affinities at the two-step isotherm ; the second step in the two-step isotherm may be corresponding to the collapse of water cluster caused by excess amount of adsorbates beyond the threshold concentration. The experimental results also supports not only two-layer and the reorientation model but also the water cluster collapse model, because the more the oxygen functional groups, the more is the starting concentration of second step shifted toward a larger concentration as observed for the nitrobenzene and phenol adsorption isotherms as depicted in Figs.3 and 5, respectively. From the experimental results and the brief numerical analysis, two-step adsorption isotherms of aromatic compounds can be explained by some models of two-layer model, molecular reorientation model, and/or water cluster collapse model so far. Further experiments will be required to inspect the adsorption mechanism in detail.

\section{Summary}

Adsorption of several aromatics of aniline, benzoic acid, nitrobenzene, phenol and bisphenol $\mathrm{A}$ in aqueous phase onto oxidized activated carbons were examined comparing with non-oxidized counterparts. Based on the experimental results, a brief numerical analysis and the related literature, the following conclusions can be deduced.

1) Langmuir isotherms can be used to represent the aromatics adsorption onto non-oxidized and oxidized activated carbons. Both adsorption capacities and affinities of aniline, benzoic acid, nitrobenzene and phenol are significantly reduced by oxidation of the activated carbons, but they are not observed for the larger molecule of bisphenol A.

2) Adsorption sites on the oxidized activated carbon are considered to be external area around the oxygen functional groups in which the kinetics is controlled by adsorbates-surface interactions, whereas those on the non-oxidized carbon will be micropore located at inner particle in diffusion controlled adsorption kinetics.

3) The $\pi-\pi$ dispersive interactions between benzene ring of aromatics and graphene sheet is considered to be predominantly operative for the adsorption onto non-oxidized activated carbons, whereas the hydrogen bonding between aromatics and surface oxygen functional groups will be working for oxidized carbons.

4) For the oxidized activated carbons, two-step isotherms can be observed for the all aromatics used. By the brief numerical analysis for the two-step adsorption isotherms, it can be estimated that the mono-layer adsorption in the flat-on position will take place for the first step, and not only the two-layer model but also the molecular reorientation model switching from flat-on to end-on position and/or the water cluster collapse model can be possible for the second step isotherms.

\section{Acknowledgements}

The authors thank Calgon Mitsubishi Chemical Corporation for providing the granular activated carbons. They are also grateful to Dr. Masami Aikawa of Kisarazu National College of Technology and Ms. Yoko Fujimura of Chiba Prefectural Environmental Research Center, Japan, for their discussion and suggestion on the study.

\section{References}

1) E. Todaka, K. Sakurai, H. Fukata, H. Miyagawa, M. Uzukı, M. Omorı, H. Osada, Y. Ikezukı, O. Tsutsumi, T. Iguchi and C. Morı, Environ. Res. 99 (2005) 195-203.

2) S. Safe, Trends in Endocrinology and Metabolısm 16 (2005) 139-144.

3) M. Machida, Y. Kikuchi, M. Aikawa and H. Tatsumoto, Colloids Surf., A : Physicochem. Eng. Aspects 240 (2004) 179-186.

4) A. Uçer, A. Uyanık and Ş. F. Aygün, Sep. Purif. Technol. 47 (2006) 113-118.

5) Y. Kıkuch1, Q. Q1an, M. Machida and H. Tatsumoto, Carbon 44 (2006) 195-202.

6) Y. Kato, M. Machida, Q. Q1an and H. Tatsumoto, TANSO 2006 [No.223] 215-219 [1n Japanese].

7) D. M. Nevska1a, A. Santianes and V. Muñoz, Carbon 37 (1999) $1065-$ 1074.

8) Y. Fu, R. S. Hansen and F. E. Bartell, J. Phys. Chem. 53 (1948) 374386.

9) R. S. Hansen, Y. Fu and F. E. Bartell, J. Phys. Chem. 53 (1949) 769785 .

10) C. H. Giles, T. H. MacEwan, S. N. Nakhwa and D. Smith, J. Chem. Soc. (1960) 3973-3993.

11) R. W. Couphlin and F. S. Ezra, Environ Sci. Technol. 4 (1968) 291297.

12) M. Machida, M Aikawa and H. Tatsumoto, J. Hazard. Mater. B120 (2005) 271-275.

13) M. Machida, T. Mochimaru and H. Tatsumoto, Carbon 44 (2006) 2681-2688.

14) M. Mofarahi, M. Sadramelı and J. Towfighi, J. Chem. Eng. Data 48 (2003) 1256-1261.

15) H. P. Boehm, Carbon 32 (1994) 759-769.

16) C. O. Ania and T. J. Bandosz, Langmuir 21 (2005) 7752-7759.

17) H. Marsh and R. Menendez, Fuel Process. Technol. 20 (1988) 269296.

18) P J. F. Harris, Crit. Rev. Solid State Mater. Sci. 30 (2005) 235-253.

19) N. Yoshizawa, TANSO 2006 [No.221] 25-30 [in Japanese].

20) J. Qi, Z. Li, Y. Guo and H. Xu, Mater. Chem. Phys. 87 (2004) 96-101.

21) C. -T. Hsieh and H. Teng, Carbon 38 (2000) 863-869.

22) A. P. Terzyk, J. Colloud Interface Sci. 268 (2003) 301-329.

23) M. Franz, H. A. Arafat and N. G. Pinto, Carbon 38 (2000) 18071819.

24) P. M. Álvarez, J. F. García-Araya, F. J. Beltrán, F. J. Masa and F. Medina, J. Colloıd Interface Sci 283 (2005) 503-512.

25) J -S. Bae and D. D. Do, Chem. Eng. Scl. 57 (2002) 3013-3024.

26) Q. Gan, S. J. Allen and R. Matthews, Waste Manage. 24 (2004) 841848.

27) M P. Sorıaga and A. T. Hubbard, J. Amer. Chem. Soc. 104 (1982) 2735-2742. 